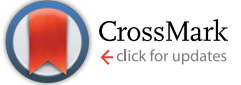

Cite this: J. Mater. Chem. A, 2016, 4, 2484

Received 12th November 2015

Accepted 5th January 2016

DOI: $10.1039 / \mathrm{c} 5 \mathrm{ta0} 0166 \mathrm{~g}$

www.rsc.org/MaterialsA

\section{"Nanotraps" in porous electrospun fibers for effective removal of lead(II) in water}

\author{
Anitha Senthamizhan, t $^{* a}$ Brabu Balusamy, t $^{a}$ Asli Celebioglu ${ }^{a}$ and Tamer Uyar*ab
}

\begin{abstract}
Here, we have put in conscientious effort to demonstrate the careful design of binding sites in fibers and their stability for enhanced adsorption of metal ions, which has proven to be a challenging task until now. Dithiothreitol capped gold nanoclusters (AuNCs) are successfully encapsulated into a cavity in the form of pores in electrospun porous cellulose acetate fibers (pCAFs) and their assembly creates a "nanotrap" for effective capture of $\mathrm{Pb}^{2+}$. The enhanced immobilization capacity of AuNCs into the interiors of the fibers and their non-aggregated nature offer enhanced adsorption sites, thus reaching maximum extraction capacity up to $1587 \mathrm{mg} \mathrm{g}^{-1}$ for $\mathrm{Pb}^{2+}$. The remarkable finding from this approach has shown that the diffusion of $\mathrm{Pb}^{2+}$ into the interiors of the AuNC encapsulated porous cellulose acetate fiber ( $p C A F / A u N C$ ) is in line with the penetration depth of AuNCs. The effectiveness of the pCAF/ AuNC has been compared with that of the AuNC decorated non-porous cellulose acetate fibers (nCAF/ AuNC). The findings have shown a remarkable improvement in the adsorption efficiency by increasing the availability and stability of adsorption sites in the pCAF/AuNC. We strongly believe that the proposed approach might provide a new insight into developing nanotraps to eliminate the usual limitations including denaturation of adsorbents on supported matrices.
\end{abstract}

\section{Introduction}

Rapid development of global industrial activities has caused the increased release of heavy metal ions into water bodies, affecting human health and environmental safety due to their toxic and carcinogenic nature even at a very low concentration. ${ }^{1-3}$ The removal of toxic metal ions and accessibility of clean water remain a critical challenge to mankind in the current scenario, expected to increase in the near future. This has necessitated the need for effective removal of toxic metal ions. Until now, various techniques have been adopted for the successful removal of heavy metal ions from polluted water, which include membrane separation, chemical precipitation, ion exchange, reverse osmosis, adsorption and so forth. ${ }^{4-8}$ Precisely, adsorption is considered to be the most effectual method owing to its low cost, good performance, and troublefree operation, and its ability to use a variety of materials as an adsorbent. ${ }^{9-13}$ Although nanoparticles play an active role in removing these metal ions, their practical applicability in waste water treatment is limited due to the need for additional

\footnotetext{
${ }^{a}$ UNAM-National Nanotechnology Research Center, Bilkent University, Ankara, 06800, Turkey.E-mail:uyar@unam.bilkent.edu.tr; senthamizhan@unam.bilkent.edu.tr

${ }^{b}$ Institute of Materials Science \& Nanotechnology, Bilkent University, Ankara, 06800, Turkey

† Electronic supplementary information (ESI) available: BET results, SEM images, XPS spectra, photographs, UV spectra and EDX mapping. See DOI: $10.1039 / \mathrm{c} 5$ ta09166g

\$ These authors contributed equally to the research.
}

separation procedures to recycle them from water. ${ }^{14-17}$ In order to evade these issues, supported matrices have been introduced, due to which electrospun fibers have emerged as a successful family of sorbents for removing heavy metal ions in the recent past. $^{18-23}$

The most critical problem encountered in adsorbents including nanofibers is their limited adsorption capacity due to the adsorption of metal ions on their surface as monolayers and denaturation of adsorbents. It is known that the adsorption behavior is based on the functional groups present on the surface of adsorbents, which form a complex with heavy metal ions. Therefore, the adsorbent is expected to showcase high porosity, large surface area, and strong binding-site accessibility for adsorbates to result in rapid removal and high adsorption capacity. ${ }^{24-30}$ As an example, the recent study by Zhenghui Li et al. has proved the advantage of porous structures for excellent adsorption of organic pollutants in water. ${ }^{31}$ The recent past has seen extensive research on the interaction of toxic pollutants with monolayer-protected metal nanoclusters (MNCs) for the advanced development of better sensing procedures. This has seen the emergence of MNCs to act as an efficient platform for new generation sensing tools. ${ }^{32-36}$

The performance of the sensor is solely based on the changes in the properties of the gold core or the monolayer. In both cases, the stability of MNCs forms the crux due to their sensitivity towards the environment. Recently, we have devised an effective method for integrating MNCs with nanofibers and further studying their sensing performance. ${ }^{37-39}$ The rapid 
adsorption ability of the metal ions on the surface of the MNC instigated us to further study the removal performance of metal ions from water. However, a major setback in achieving this goal is the protection of the functionalities of the MNC. The last few years have seen various studies for the decoration of the nanoparticles onto an electrospun fiber surface for various applications including sensors. ${ }^{40-47}$ Such a case usually includes the incorporation of nanoparticles into fibers or decorating them on the surface as a monolayer or multilayer resulting in nanoparticle/polymer composites. Interestingly, the incorporation of the nanoparticles inside the polymer fiber leads to protection of their stability while their concrete performance might be degraded. ${ }^{48,49}$ In order to overcome this circumstance, the particles are decorated on the outer surface of the fibers, resulting in enhanced performance, while the protection of nanoparticles against the environment is under question.

In our case, the presence of ligands in the nanoclusters plays a vital role in binding with target metal pollutants. Under the condition where the nanoclusters are decorated on the fiber surface, the rate of exposure is found to be higher and the ligands on the gold surface are subjected to environmental degradation, which might be expected to reduce removal efficiency. Additionally, the aggregation of MNCs also has an impact on the removal performance owing to limited interactions with the pollutant at the surface level. Thus, special attention needs to be paid to shield the MNCs against the environment because of their sensitive surface. It is a general assumption that adsorbed metal ions found on the MNCs disable them by either removing the ligands or changing their chemical nature upon interaction with metal ions. Such cases prove aggregation of nanoclusters to be unavoidable, leading to decreased adsorption performance. Based on the aforementioned points, we have worked towards finding a system to protect MNCs on their fiber surface for easy accessibility. Concern has also been put on that the selected MNCs should not lose their activity upon interaction with metal ions. Based on the literature, parameters for achieving high adsorption capacity include a large surface area, porous structure, enhanced accessibility of functional groups to the pollutants and stability. Over the last few decades, the electrospinning approach has attracted a great deal of attention due to its simplicity, cost effectiveness, feasibility and scalability for production of nanofibers from diverse materials. ${ }^{50-52}$ Electrospun fibers possess several unique properties including a high surface area, porous structure and flexibility which make them more attractive in a variety of applications such as sensors, adsorbents, catalysts, filters and tissue regeneration. ${ }^{53-56}$ We strongly believe that these criteria thoroughly match the requirement of the current study.

This research work highlights our preliminary efforts to devise a synthetic strategy for the effective removal of $\mathrm{Pb}^{2+}$ in water by creating stable "nanotraps" in electrospun porous cellulose acetate fibers (pCAFs). The resultant nanotraps enable higher uptake capacities of $\mathrm{Pb}^{2+}$ up to $1587 \mathrm{mg} \mathrm{g}^{-1}$.

\section{Experimental section}

\section{Materials}

Tetrachloroauric acid trihydrate $\left(\mathrm{HAuCl}_{4} \cdot 3 \mathrm{H}_{2} \mathrm{O}\right)$, dichloromethane (DCM, $\geq 99 \%$ (GC)), methanol ( $\geq 99.7 \%$ (GC)), acetone ( $\geq 99 \%$ (GC)), cellulose acetate, (CA, $M_{\mathrm{w}}: 30000 \mathrm{~g} \mathrm{~mol}^{-1}, 39.8$ wt $\%$ acetyl), zinc acetate dihydrate $(\sim 98 \%)$, lead(II) nitrate (99.0\%), manganese(II) acetate tetrahydrate (99\%) and nickel(II) acetate tetrahydrate $(99.0 \%)$ were procured from Sigma-Aldrich. 1,4-Dithiothreitol (DTT), cadmium nitrate tetrahydrate and mercury(II) acetate were obtained from VWR Chemicals, Fluka and Merck, respectively. All chemicals were used as received without any further purification. A Millipore Milli-Q Ultrapure Water System was used to acquire deionized water.

\section{Electrospinning of porous and nonporous cellulose acetate fibers}

The electrospun porous cellulose acetate fibers (pCAFs) and nonporous cellulose acetate fibers (nCAFs) were prepared by following the method reported earlier. ${ }^{57}$ The process involves the preparation of homogenous polymer solution $(10 \% \mathrm{w} / \mathrm{v})$ by dissolving cellulose acetate (CA) in a binary solvent mixture of DCM and acetone at $1: 1$ volume ratio. In a similar fashion, nCAFs were fabricated from the CA polymer solution which was prepared by dissolving CA at $12 \%(\mathrm{w} / \mathrm{v})$ in a DCM and methanol binary solvent mixture $(4 / 1 \mathrm{v} / \mathrm{v})$. The resulting polymer solution was subjected to electrospinning at a flow rate of $0.5-1 \mathrm{~mL} \mathrm{~h}^{-1}$ using a syringe pump (KDS-101, KD Scientific, USA) and a $3 \mathrm{~mL}$ syringe hold 27 gauge metallic needle as a spinneret under an optimized high voltage (10$15 \mathrm{kV}$ ) applied between the needle and grounded collector by a high-voltage power supply (Spellman, SL30, USA). Then, an aluminium foil was used to cover the grounded metal plate for collecting the PCAF and nCAF which was located at a distance of 10-12 $\mathrm{cm}$ from the needle. The electrospinning process was performed at $23{ }^{\circ} \mathrm{C}$ at $18 \%$ relative humidity in a Plexiglas box. The resulting porous cellulose acetate fibrous membrane (pCAFM) and nonporous cellulose acetate fibrous membrane (nCAFM) were further air dried in a fume hood at room temperature.

\section{Synthesis of DTT capped gold nanoclusters (AuNCs)}

The DTT capped gold nanoclusters (AuNCs) were prepared as described by Ding et al..$^{58}$ Briefly, $11 \mathrm{mg}$ of DTT was dissolved in $10 \mathrm{~mL}$ deionized water under vigorous stirring. This was followed by immediate addition of $768 \mu \mathrm{L}$ of $\mathrm{HAuCl}_{4}$ solution at room temperature. Then, stirring was allowed for 3 minutes, followed by the addition of aqueous sodium hydroxide to reach $\mathrm{pH}$ 8. The resulting solution was subjected to further stirring for $6 \mathrm{~h}$ to obtain a colorless solution. Under UV irradiation $\left(\lambda_{\text {ext }}-254\right.$ $\mathrm{nm}$ ), the prepared AuNC emitted red fluorescence and further the clusters will be encapsulated into pCAFs and nCAFs.

\section{Preparation of the AuNC encapsulated PCAFM and nCAFM (pCAFM/AuNC and nCAFM/AuNC)}

The method for encapsulation of AuNCs into the PCAFM and nCAFM was performed by following our previous protocols. ${ }^{59}$ 
The first step involves immersing the PCAFM and nCAFM in AuNC solution separately for $3 \mathrm{~h}$ in a shaking platform, followed by carefully removing them from the solution and subjected to drying at room temperature. Rinsing the membrane for around 15 minutes under vigorous shaking and then air drying them at room temperature remove the excess adsorbed clusters and ligands on the fiber surface.

\section{Analytical procedure for preparation of metal ion stock solution}

A stock solution $(50 \mathrm{ppm})$ of $\mathrm{Pb}^{2+}$ was prepared by dissolving lead(II) nitrate salt in deionized water. Consequently, the desired concentrations of $\mathrm{Pb}^{2+}$ were prepared by diluting an appropriate volume of the stock solution. Similarly, the same protocol was followed for solutions of $\mathrm{Cd}^{2+}, \mathrm{Zn}^{2+}, \mathrm{Ni}^{2+}, \mathrm{Mn}^{2+}$ and $\mathrm{Hg}^{2+}$.

\section{Adsorption of toxic metal ions}

Batch adsorption experiments were carried out to evaluate the removal efficiency of the pCAFM/AuNC for toxic metal ions $\left(\mathrm{Pb}^{2+}, \mathrm{Cd}^{2+}, \mathrm{Zn}^{2+}, \mathrm{Ni}^{2+}, \mathrm{Mn}^{2+}\right.$ and $\left.\mathrm{Hg}^{2+}\right)$ in water. In a typical experiment, the membrane was placed $\left(3 \mathrm{mg} \mathrm{mL}^{-1}\right)$ in a beaker containing metal ion solution $(50 \mathrm{~mL})$ followed by agitation at $100 \mathrm{rpm}$ on a mechanical shaker under ambient conditions. The effect of contact time on the removal performance of the pCAFM/AuNC for $\mathrm{Pb}^{2+}$ ions was analyzed by withdrawing the samples at an arbitrary time period, ranging from 0-24 h, from a solution concentration of $1 \mathrm{ppm}$ and $5 \mathrm{ppm}$, respectively. The pCAFM was used as the control for comparing the removal efficiency. Finally, similar conditions were followed for investigating the effect of concentration ( $50 \mathrm{ppb}$ to $5 \mathrm{ppm}$ ) on the removal performance. A similar procedure was carried out for the nCAFM/AuNC also.

The removal efficiency and adsorption capacity $\left(q_{\mathrm{e}}, \mathrm{mg} \mathrm{g}^{-1}\right)$ of the pCAFM/AuNC were calculated using the following equations.

$$
\begin{aligned}
& \text { Removal efficiency }(\%)=\frac{\left(C_{0}-C_{\mathrm{e}}\right)}{C_{0}} \times 100 \\
& \text { Adsorption capacity }\left(q_{\mathrm{e}}\right)=\frac{\left(C_{0}-C_{\mathrm{e}}\right) \times V}{m}
\end{aligned}
$$

$q_{\mathrm{e}}$ is the adsorption amount $\left(\mathrm{mg} \mathrm{g}^{-1}\right), C_{0}$ and $C_{\mathrm{e}}$ are the concentration of metal ions in the aqueous solution before and after the adsorption, respectively $\left(\mathrm{mg} \mathrm{L}^{-1}\right), V$ is the volume of the solution (L), and $m$ is the weight of the pCAFM/AuNC (g).

\section{Instrumentation}

A transmission electron microscope (TEM, Tecnai G2 F30) equipped with an EDS was employed to study the morphological and porous nature of the fibers. STEM-EDX (scanning transmission electron microscopy-energy dispersive X-ray analysis) was used to examine gold nanoclusters and metal ions on the surface of fibers. The removal efficiencies of the nanofibers were determined by measuring the residual concentration of metal ions using inductively coupled plasma mass spectroscopy (ICP-MS, Thermo, X Series II). X-ray photoelectron spectroscopy (XPS, Thermo K-alpha-monochromated) was used to confirm the adsorbed metal ions on the surface of fibers and their chemical state. Contact angle measurements were carried out using a contact-angle meter (OCA 30, Dataphysics). A UV-Vis-NIR spectrophotometer (Cary 100) was used to record the absorbance spectra. The surface area and pore size of the fibrous membrane were obtained using a Brunauer, Emmett and Teller (BET) surface area analyzer (Micromeritics TriStar 3000). The data were collected at $77 \mathrm{~K}$ in the range of 0.1-0.9 relative pressure.

\section{Results and discussion}

The present study aims at designing a method for creating nanotraps by encapsulating dithiothreitol capped gold nanoclusters (AuNCs) with an average size of 2-3 $\mathrm{nm}$ into the electrospun porous cellulose acetate fibers (pCAFs), named as pCAF/AuNC and enhancing more active sites by loading a higher amount of AuNCs into the porous fibers. In this approach, we have fabricated pCAFs (diameter $\sim 1-1.5 \mu \mathrm{m}$ ) with an average pore size of 30-40 $\mathrm{nm}$ using a simple and versatile single step electrospinning technique by using the solvents and their composition of DCM/acetone $(1 / 1(\mathrm{v} / \mathrm{v}))$. The attained porous structure in pCAFs might be due the rapid evaporation of the highly volatile solvents (DCM and acetone) as compared with nCAFs, with consequent solidification of polymer chains as reported by our earlier publication. ${ }^{57}$ To the best of our knowledge, no effort has been put on the metal ion removal performance, although the sensing characteristics of AuNCs are well known. ${ }^{\mathbf{5 8 , 5 9}}$ Interestingly, we have been motivated by the specific characteristic of DTT to form a stable complex with metal ions using both of its sulfur donors. ${ }^{\text {60-62 }}$ When the pCAFs are immersed into the AuNC solution, the pores in the fibers get swelled up, facilitating the transportation of AuNCs into the fiber interior. Further, they can effectively bind on the pore walls by the formation of hydrogen bonding between them.

The excess amount of unbound clusters on the fiber surface is carefully washed out immediately after drying the membrane. This is done to prevent the blockage of pores which might hamper the penetration of analytes into the fiber interiors. Also, it is believed that the diffusion of AuNCs inside the pCAF facilitates further entry of AuNCs from the solution into the interior of the porous fiber. The TEM image of the pCAF/AuNC highlights the penetration of AuNCs into the interior of the pCAF up to $\sim 300 \mathrm{~nm}$ in depth as depicted in Fig. 1a, wherein the nanoclusters are well dispersed rather than aggregates on the external surface of the fibers. Expectedly, a cavity in the form of the fiber pores protects the properties of AuNCs against the environment for a prolonged time period. Also, we can observe the uniform penetration of the encapsulated AuNCs into the fiber matrix, firmly anchored on their surface which can act as nanotraps for capturing toxic metal ions in water as demonstrated in Fig. 1a and b. The inset in Fig. 1b represents the high resolution TEM image of the AuNC showing a lattice spacing of $0.235 \mathrm{~nm}$ which corresponds to the (111) lattice plane of the 

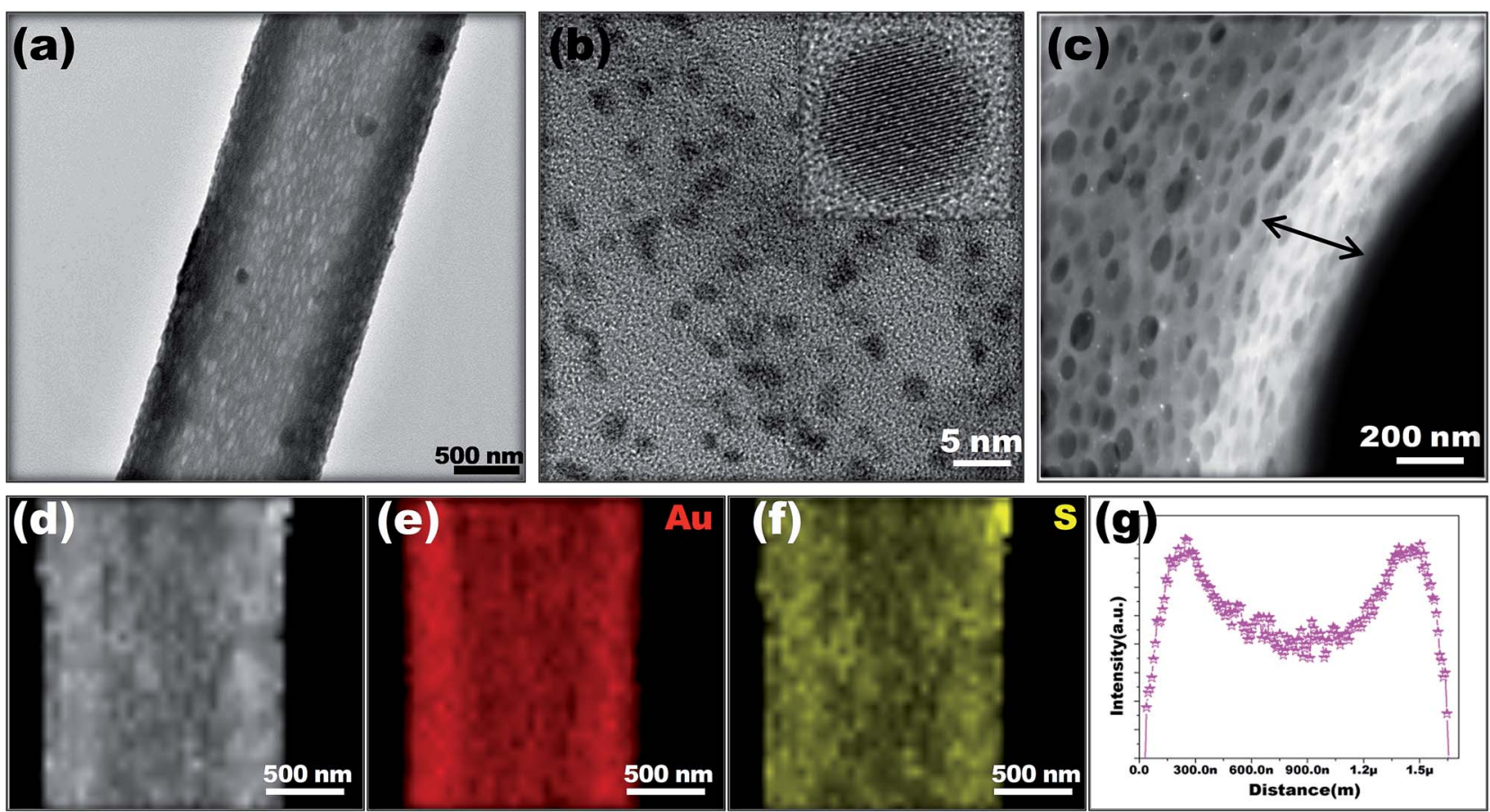

Fig. 1 (a) TEM image of the gold nanocluster (AuNC) encapsulated porous cellulose acetate fiber (pCAF/AuNC). The image clearly demonstrates that the penetration depth is almost uniform throughout the fiber, confirming the deep penetration of AuNCs into the fiber interior. (b) TEM image of AuNCs. The inset shows AuNCs having a lattice spacing of $0.235 \mathrm{~nm}$ which corresponds to the (111) lattice plane of the face-centered cubic (fcc) gold. ( $c$ and d) HAADF-STEM image and EDS elemental mapping of (e) Au and (f) S. The image confirms that the porous nature of the fiber is highly persistent and does not degrade following the incorporation of AuNCs. (g) EDS intensity line profile of Au taken across the pCAF/ AuNC surface.

face-centered cubic (fcc) gold. ${ }^{63}$ The STEM image of the pCAF/ AuNC confirms that the porous nature of pCAFs is highly persistent and does not degrade following the incorporation of AuNCs which is expected to enhance the rapid diffusion and fast response with heavy metal ions as seen in Fig. 1c.

The size of the AuNC and pores in pCAFs are considered to be important factors for incorporation. It would be highly appreciated if the AuNCs are smaller than the pores for effectual incorporation, to prevent blockage of the pore wall.

The above discussed facts have aided us in optimizing the dipping time as $3 \mathrm{~h}$ for the successful incorporation of AuNCs into pCAFs under a shaker. The uniform distribution of AuNCs in the PCAF is confirmed by STEM-EDX elemental mapping as depicted in Fig. 1d-f. The line intensity profile in Fig. $1 \mathrm{~g}$ further confirms that the penetration of AuNCs is uniform along fibers. However, a question arises on whether the creation of nanotraps will dominate the removal efficiency of toxic metal ions or not. To confirm this, non-porous cellulose acetate fibers (nCAFs) were decorated with AuNCs which are named as nCAF/ AuNC and their potency in metal ion removal was systematically compared with the pCAF/AuNC. Fig. $2 \mathrm{a}$ and c show the SEM images of nCAFs before and after decorating with AuNCs with an average diameter of $1 \mu \mathrm{M} \pm 280 \mathrm{~nm}$ and $1.3 \mu \mathrm{M} \pm 250 \mathrm{~nm}$, respectively. Compelling confirmation has been obtained from the cross-sectional observation of nCAFs about their solid nature as shown in Fig. $2 \mathrm{~b}$ and d. The energy dispersive X-ray (EDX) elemental mapping of the nCAF/AuNC demonstrates the uniform distribution of AuNCs decorated over the fibrous membrane as depicted in Fig. 2e-i. The comparative surface area and pore size of the nCAFM and PCAFM are given in Table S1. $\dagger$ The specific surface area of the nCAFM and PCAFM was determined to be 3.36 and $9.73 \mathrm{~m}^{2} \mathrm{~g}^{-1}$, respectively. The average pore size of the respective fibers was found to be 6.2 and 12.5 $\mathrm{nm}$. After encapsulating with AuNCs, the PAFM/AuNC shows a slight decrease in its surface area and pore size which were observed to be $6.89 \mathrm{~m}^{2} \mathrm{~g}^{-1}$ and $9.2 \mathrm{~nm}$, respectively.

Although the wetting characteristics of the fibrous membrane play an important role in the process of adsorption, they are not considered as a primary factor. ${ }^{64-67}$ If the fibrous membrane does not permit penetration of polluted water, the reaction takes place only on the surface of the fiber, resulting in lower adsorption capacity. As a proof of concept, a recent report well demonstrated that water-dispersibility nature of the adsorbent enhances the adsorption of alizarin red in the aqueous solution because its wettability nature facilitates the pores much more accessible to the adsorbates. ${ }^{68}$ We have paid more attention to study the interaction between fibrous mat and water and their transport behavior to improve the overall removal capacity of metal ions. Until now, limited attention has been paid to liquid moisture transport behaviors of electrospun fibrous membranes especially when they are considered for removal of metal ions. It has been observed that various surface modification processes can be used to design the wetting behavior of fibrous membranes. ${ }^{69-73}$ 

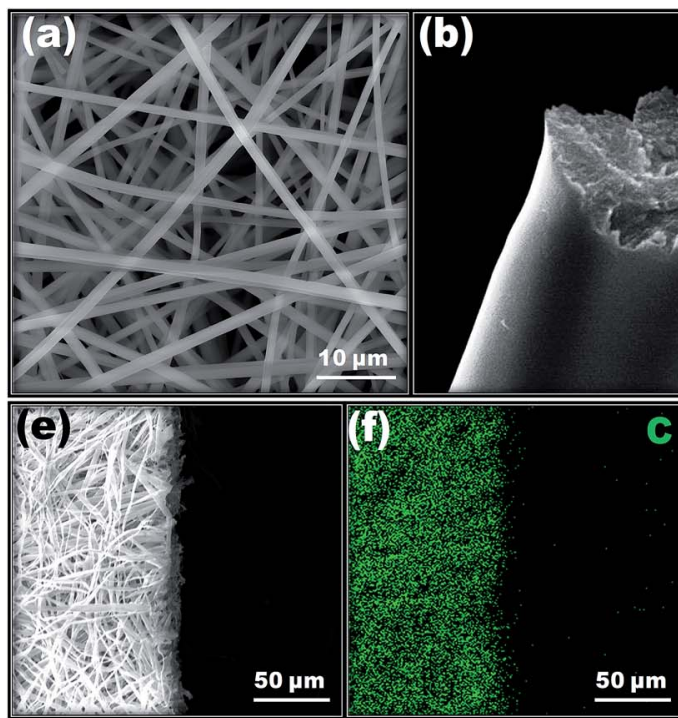
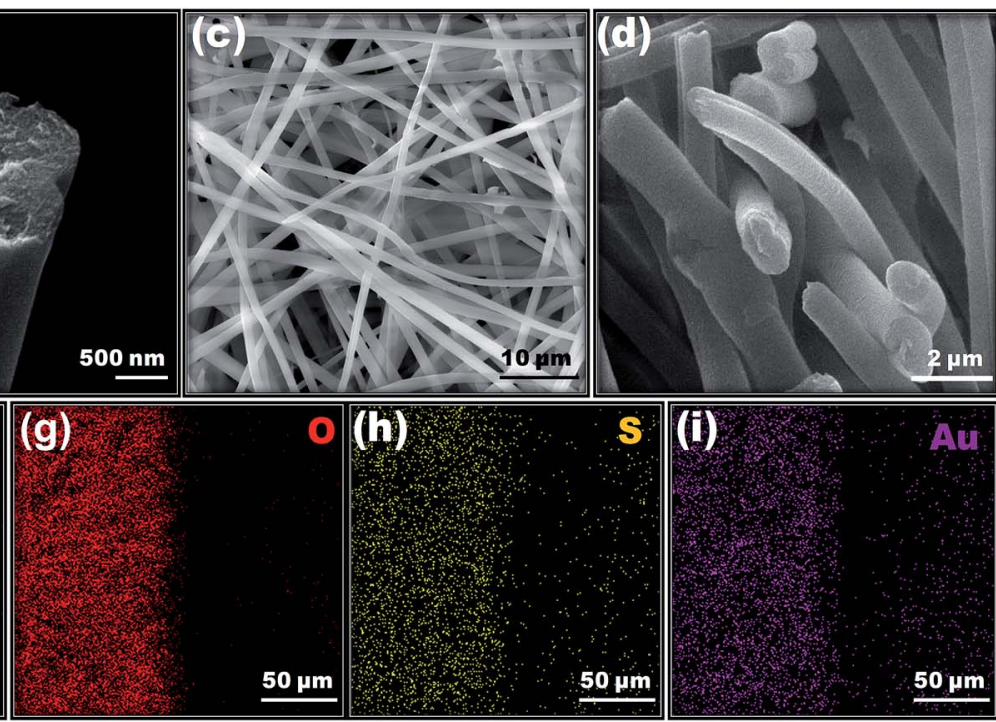

Fig. 2 SEM image and the cross-sectional view of ( $a$ and b) nCAF and (c and d) nCAF/AuNC, confirming their nonporous nature. (e) SEM image and EDX mapping of the nCAFM/AuNC ( $f$ ) $C(g) O(h) S$ and (i) Au. The uniform distribution of AuNCs decorated over the fibrous membrane is clearly seen.

Further, we are gratified at presenting the primary report here talking about the enhanced wetting characteristics and capillary action of the electrospun porous cellulose acetate fibrous membrane (PCAFM) and nonporous cellulose acetate fibrous membrane (nCAFM) by encapsulating AuNCs. In depth information on the wettability nature and the diffusion ability of water in the PCAFM and nCAFM before and after coating with AuNCs is taken from the time dependent contact angles. The contact angle of the pristine PCAFM and nCAFM is measured to be $137^{\circ}$ and $106^{\circ}$, respectively, which is found to decrease to $107^{\circ}$ and $98^{\circ}$ after 3 minutes as shown in Fig. 3a and c. It is found that roughness is the inducing factor behind the increase in the water contact angle of the pCAFM. Interestingly, it is also noticed that the contact angle of both the pCAFM/AuNC and nCAFM/AuNC suddenly drops down to zero, followed by the water droplets spreading out and permeating into the membrane, once it comes into contact with the membrane surface (see Fig. S1 $\dagger$ ). This is confirmed in Fig. $3 \mathrm{~b}$ and d, showing the capability of incorporating AuNCs modifying the surface characteristics, from hydrophobic to hydrophilic. Fig. 3e-h further demonstrate the real time wettability nature of the fibrous membrane by placing a few drops of water on its surface.

The diffusion of water droplets is uniform in all directions at a fraction of seconds whereas the water droplet is stable on the pristine membrane. The above stated fact has been clearly supported by evidence from the measured contact angle. Notably, immediate water diffusion happens in the pCAFM/ AuNC as compared with the nCAFM/AuNC, which could be attributed to a stronger capillary effect induced by the large volume of pores present in the fibers. This further confirms that the porous structure is well maintained post-decorating AuNCs. On exposure to UV light ( $\lambda_{\text {ext }}-366 \mathrm{~nm}$ ), both AuNCs and fluorescein isothiocyanate (FITC) dye emit red and green fluorescence, respectively. Thus, we have combined these efficient attributes to perform an experiment for demonstrating the capillary motion induced liquid transport behavior in the pCAFM/AuNC and nCAFM/AuNC as illustrated in Fig. 4. In addition, the fast propagation of water in the PCAFM/AuNC is supported by Video S1.†

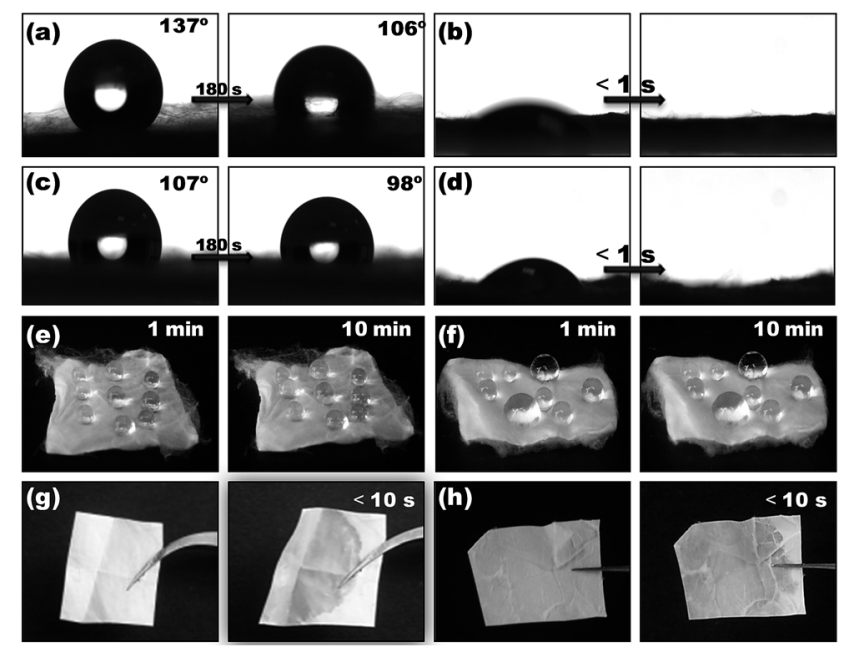

Fig. 3 Wetting behavior of water on porous and nonporous cellulose acetate fibrous membranes before and after encapsulation of AuNCs. Contact angle of the (a) pCAFM (b) pCAFM/AuNC (c) nCAFM, and (d) nCAFM/AuNC. The contact angle of both the pCAFM/AuNC and nCAFM/AuNC suddenly drops down to zero within seconds, followed by the water droplets spreading out and permeating into the membrane. The water droplet was set to $5 \mu \mathrm{L}$ during the contact angle measurement. Photograph of the water droplet on the (e) pCAFM, ( $f$ ) nCAFM, (g) pCAFM/AuNC and (h) nCAFM/AuNC. The wet zone in the pCAFM/AuNC and nCAFM/AuNC represents the spreading of water droplets. 


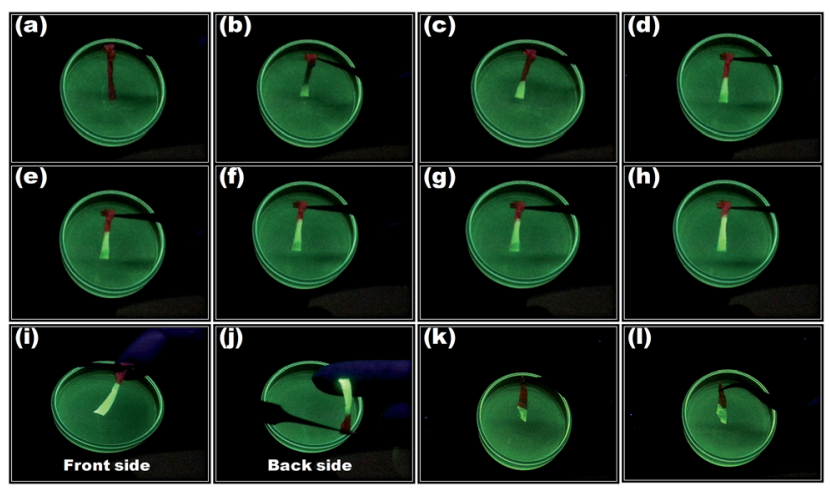

Fig. $4(a-h)$ A sequence of frames showing the propagation of water upward in the pCAFM/AuNC strip. The red and green color represents the characteristics of AuNCs and FITC dye. (i \& j) Front and back sides of the pCAFM/AuNC after spread out of water. The water is efficiently diffused into the membrane, confirmed from the back side of the membrane emitting green fluorescence due to the presence of transported FITC dye. $(k \& l)$ Photographs of the nCAFM/AuNC showing the propagation of water after $30 \mathrm{~s}$ and $2 \mathrm{~min}$, respectively.

The experiment started with dipping a piece of pCAFM/AuNC strip in water containing FITC dye, and transport of water was monitored under UV light. Captivating photographs within 10 seconds of reaction time highlighted the propagation of water upward in the pCAFM/AuNC, comprehensibly demonstrating the green emission of FITC as in Fig. 4a-h. Remarkably, the diffusion has been found to be uniform on the surface and the bottom of the fibers, which is confirmed from the back side of the membrane emitting green fluorescence due to the presence of transported FITC dye (Fig. $4 \mathrm{i}$ and $\mathrm{j}$ ). It is interesting to note that the transport rate of water containing FITC dye in the pCAFM/AuNC is significantly higher than that in the nCAFM/ AuNC (Fig. 4k and 1). Also, limitability is observed in the transport of FITC dye in the nCAFM/AuNC to an extent. Even after a prolonged period of time, no markable diffusion of water was noticed. Significant improvement is expected from the nanoporous channels to greatly increase the capillary motion and aid water transport. Hence, this proves the ability of the porous structure of fibers to determine their wetting and transport nature. As seen from the experiment, it is understood that the immersion of the membrane in water did not alter the integrity of the membrane structure. The anchored AuNC is found to tightly bind on the wall of the pore, combined with the formation of a strong concrete wall making it stable against swelling. Initially, the adsorption efficiency of the pCAFM/AuNC was evaluated against various toxic metal ions such as $\mathrm{Pb}^{2+}$, $\mathrm{Ni}^{2+}, \mathrm{Mn}^{2+}, \mathrm{Cd}^{2+}, \mathrm{Zn}^{2+}$ and $\mathrm{Hg}^{2+}$, at $1 \mathrm{ppm}$ concentration as shown in Fig. 5a. Interestingly, the pCAFM/AuNC has the ability to remove the metal ions in the order of $\mathrm{Pb}^{2+}>\mathrm{Zn}^{2+}>\mathrm{Cd}^{2+}>$ $\mathrm{Hg}^{2+}>\mathrm{Ni}^{2+}>\mathrm{Mn}^{2+}$. The results showed a higher degree of removal efficiency towards $\mathrm{Pb}^{2+}$ when compared to other metal ions. Thus, this shifted our focus towards investigating about the feasibility of using designed nanotraps as an adsorbing substrate against the removal of $\mathrm{Pb}^{2+}$ in water. The removal efficiency as a function of contact time has been studied and is shown in Fig. 5b. The pCAFM has been used as a control. The resulting outcome suggests the removal of $\sim 70 \%$ of $\mathrm{Pb}^{2+}$ within 5 minutes, and the removal efficiency gradually increases and reached $99 \%$ within $3 \mathrm{~h}$.

The obtained result prompted us to keep $3 \mathrm{~h}$ as the contact time for the following experiments to achieve complete equilibrium. At low concentrations, it is presumed that the ideal adsorbent will remove the metal ions. In addition to the high adsorption capacity in a relatively high concentration range, the pCAFM/AuNC also exhibits an excellent adsorption performance in lower concentration ranges from $50 \mathrm{ppb}$ as illustrated in Fig. 5c. At this concentration, $99 \%$ of $\mathrm{Pb}^{2+}$ is removed from the water. The removal efficiency as a function of $\mathrm{Pb}^{2+}$ concentration represents a decrease in the adsorption capacity with increasing $\mathrm{Pb}^{2+}$ concentration. The comparative removal efficiency of the pCAFM/AuNC with the nCAFM/AuNC is measured and presented in Fig. 5c. At a lower concentration, it is observed that the nCAFM/AuNC exhibits good ability for removing the metal ions because the process might be due to surface adsorption. An increase in the concentration from 1 ppm to 5 ppm notices a solid efficiency decrease, due to limited adsorption sites available for uptake of metal ions.

The resulting outcome has proved that the pCAFM/AuNC shows higher uptake capabilities than the nCAFM/AuNC. The adsorption capacity of the pCAFM/AuNC for $\mathrm{Pb}^{2+}$ has been studied and the results are shown in Fig. 5d. The enhanced uniform encapsulation of AuNCs inside the porous structure and their active interaction with metal ions lead to maximum adsorption capacity of $\mathrm{Pb}^{2+}$ which is found to go as high as 1587 $\mathrm{mg} \mathrm{g}^{-1}$. Here, we list down the reasons behind the ability of the pCAFM/AuNC to exhibit such high adsorption capacity. Firstly, the ability of the porous structure to showcase high loading encapsulation of non-aggregated AuNCs, resulting in improved interaction with metal ions. Secondly, the well maintained nanopore channels post-encapsulation efficiently facilitate better water permeation without losing their physical structure. And further, a large surface area, abundant inter accommodations and well dispersed AuNCs in nanopores form a nanotrap which provides increased active sites for adsorption. The discussed method has also shown that the diffusion of $\mathrm{Pb}^{2+}$ into the pCAF/AuNC is equivalent to the penetration depth of AuNCs as clearly shown in Fig. 6a. The metal ions are found to have a strong bond with DTT, which binds to the gold surface through Au-S bonds. ${ }^{74,75}$

When the membrane comes into contact with the water, the metal ions are rapidly penetrated and adsorbed on the surface of AuNCs as confirmed by the EDS line profile of $\mathrm{Au}, \mathrm{S}$, and $\mathrm{Pb}$ as clearly seen in Fig. S2. $\uparrow$ The TEM image of the pCAF/AuNC surface after adsorption of $\mathrm{Pb}^{2+}$ evidenced that the adsorption did not induce the aggregation of AuNCs (see Fig. 6b). The morphology of the pCAFM/AuNC after adsorption of $\mathrm{Pb}^{2+}$ has been studied and the results reveal the formation of crystals on the surface when the concentration reaches $50 \mathrm{ppm}$ as illustrated in Fig. $6 \mathrm{c}$ and d. The EDX spectra and mapping of formed crystals are shown in Fig. 6e. The interaction of AuNCs upon addition of different concentrations of $\mathrm{Pb}^{2+}$ (50 ppb, 1 ppm, 10 ppm, 20 ppm and $50 \mathrm{ppm}$ ) has been studied by UV-Vis spectra and their fluorescence characteristics have been observed under 
(a)

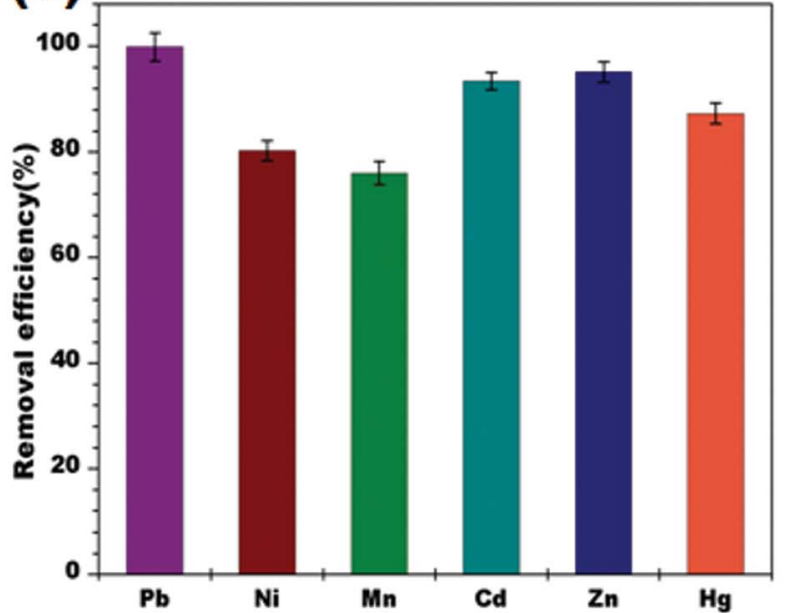

(c)

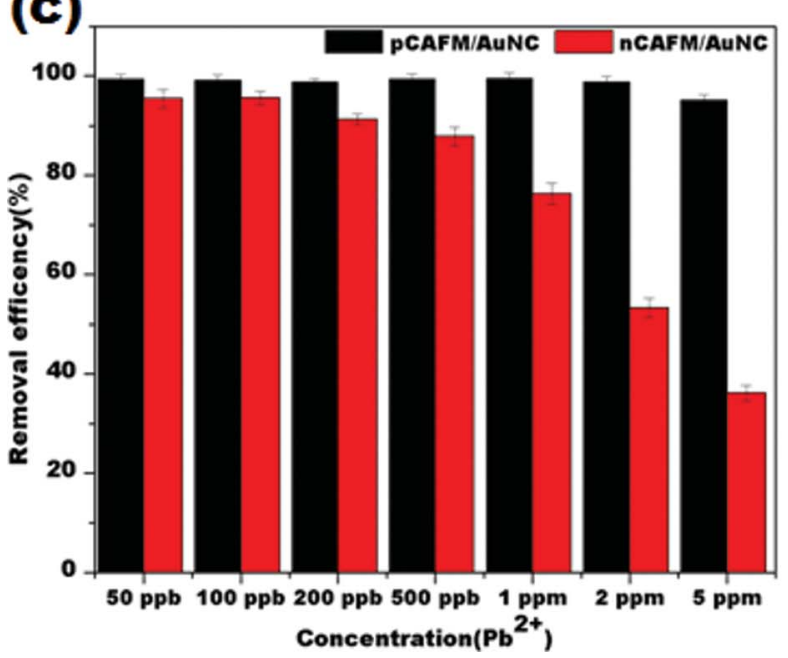

(b)

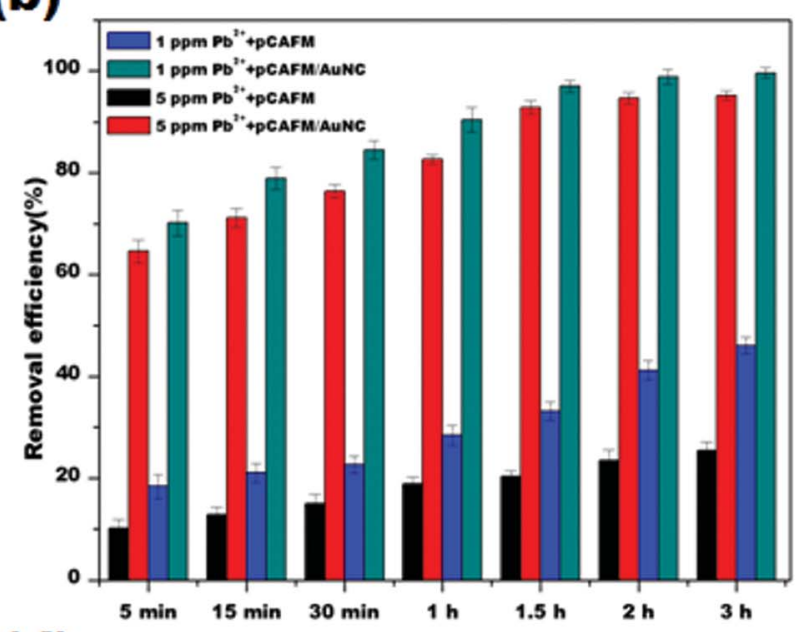

(d) Time

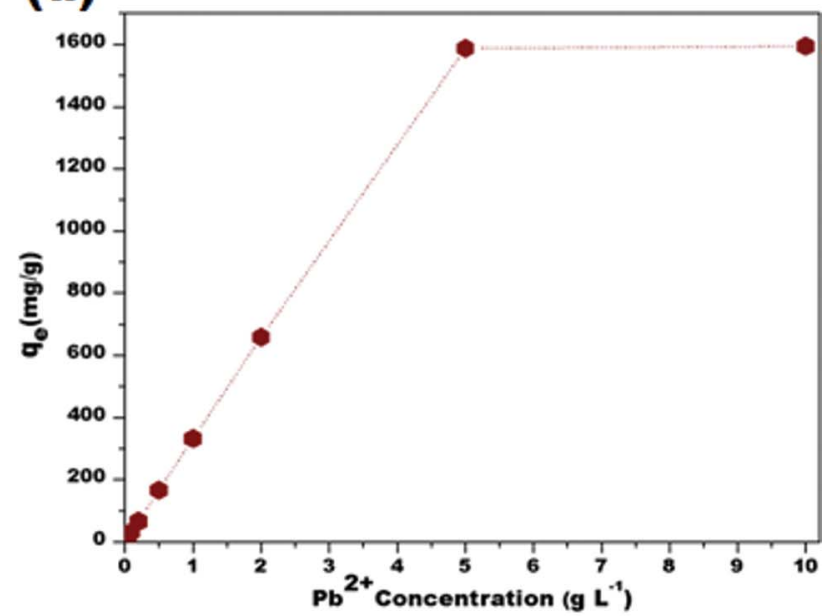

Fig. 5 (a) Removal efficiency of toxic metal ions $\left(\mathrm{Pb}^{2+}, \mathrm{Ni}^{2+}, \mathrm{Mn}^{2+}, \mathrm{Cd}^{2+}, \mathrm{Zn}^{2+}\right.$ and $\left.\mathrm{Hg}^{2+}\right)$ using the pCAFM/AuNC in water. The pCAFM/AuNC has the ability to remove the metal ions in the order of $\mathrm{Pb}^{2+}>\mathrm{Zn}^{2+}>\mathrm{Cd}^{2+}>\mathrm{Hg}^{2+}>\mathrm{Ni}^{2+}>\mathrm{Mn}^{2+}$. The concentration is set to $1 \mathrm{ppm}$ for all metal ions. (b) Time dependent removal efficiency of $\mathrm{Pb}^{2+}$ at $1 \mathrm{ppm}$ and $5 \mathrm{ppm}$ concentrations. (c) Effect of initial concentration of $\mathrm{Pb}^{2+}$ on removal efficiency of the pCAFM/AuNC and its comparative removal efficiency with the nCAFM/AuNC. (d) Adsorption capacity for the pCAFM/AuNC.

UV light (shown in the inset in Fig. S3†). Precisely, it is noticed that no change is observed at low concentrations, while there is an emergence of a new peak at around $280 \mathrm{~nm}$ corresponding to the strong complexation between DTT and $\mathrm{Pb}^{2+}$. Expectedly, there was nil fluorescence decrease with any of the tested concentrations. However, on adding $50 \mathrm{ppm}$ solution to the AuNC solution, the bottom of the vial showed the formation of a precipitate owing to strong complex formation between $\mathrm{Pb}^{2+}$ and DTT. As can be seen from Fig. S3, $\dagger$ even after the formation of the precipitate, the AuNC emits red fluorescence revealing the unaffected nature of the AuNC by $\mathrm{Pb}^{2+}$. This is found to be the underlying mechanism for the observed adsorption efficiency for $\mathrm{Pb}^{2+}$ ions, because a similar type of crystal formation has not been seen with any other metal ions even at a higher concentration as seen in Fig. S4. $\dagger$

The information on the chemical state of the pCAFM/AuNC ( $\mathrm{S}$ from DTT and Au from the AuNC) before and after adsorption of $\mathrm{Pb}^{2+}$ has been studied using XPS spectra as shown in Fig. S5a and b. $\dagger$ The observed two peaks at $162.5 \mathrm{eV}$ and $163.7 \mathrm{eV}$ correspond to the $\mathrm{S}-\mathrm{Au}$ covalent bond, confirming the successful preparation of thiol bound gold nanoclusters and free $\mathrm{SH}$ groups. ${ }^{76}$ Upon interaction with $\mathrm{Pb}^{2+}(10 \mathrm{ppm})$, there is no change in the chemical state of the $\mathrm{S}$ and Au. The adsorbed $\mathrm{Pb}^{2+}$ has been confirmed by taking XPS spectra on the PCAFM/ AuNC surface (Fig. 6f). Further, adsorption of other metal ions on the pCAFM/AuNC surface was also analyzed by XPS (Fig. S6†). In addition, EDX mapping (Fig. S7†) confirms the uniform adsorption of metal ions over the membrane surface owing to the formation of metal ion complexes with DTT molecules since they contain donor groups which are highly capable of making complexes. The detailed investigation confirms that the formation of nanotraps and their ability to capture toxic pollutants inside their cavity make up for an environmentally safe adsorbent. 

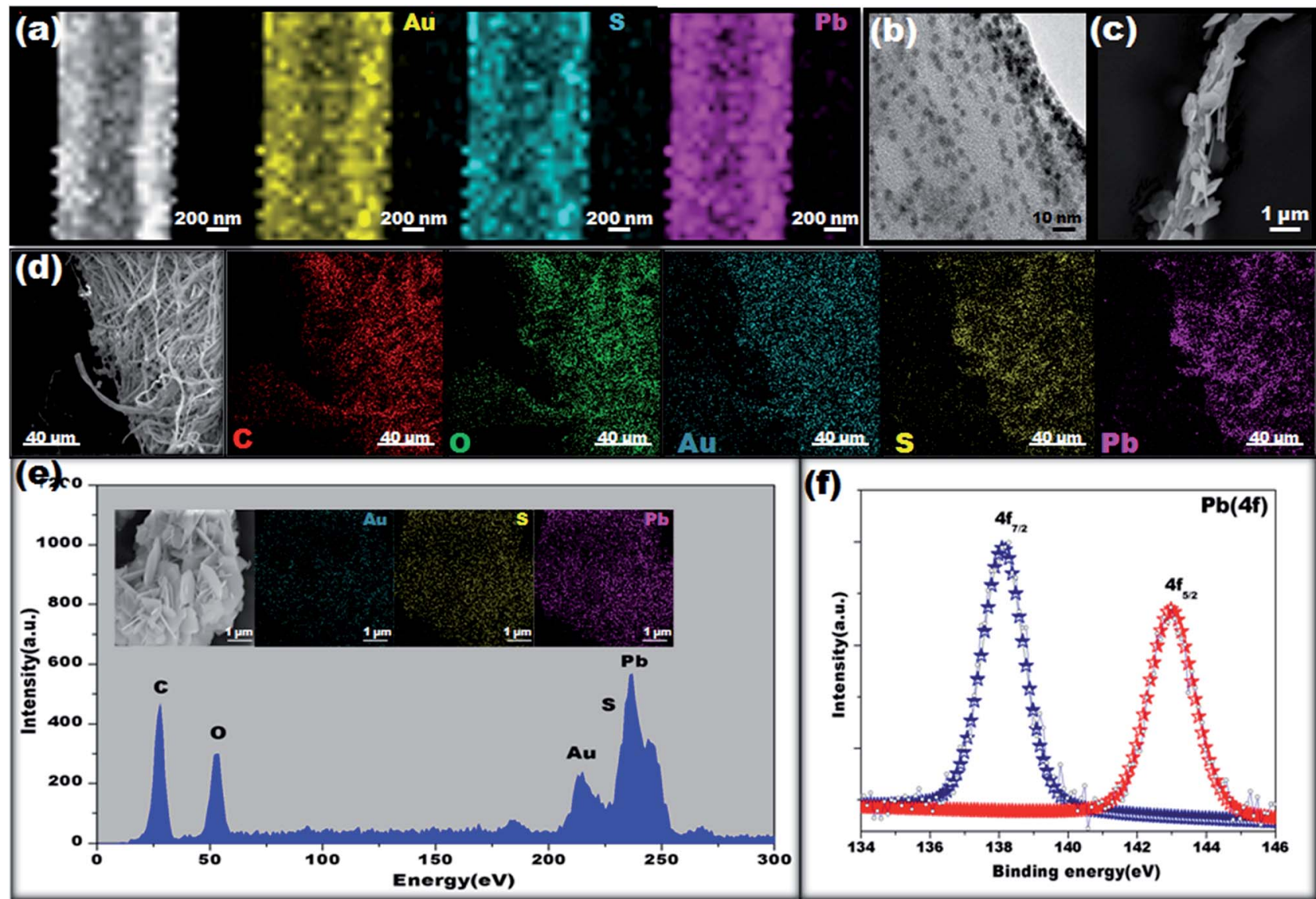

Fig. 6 Investigation on $\mathrm{Pb}^{2+}$ binding interaction. (a) HAADF-STEM images and corresponding elemental mapping images of the pCAF/AuNC after treatment with $10 \mathrm{ppm} \mathrm{Pb}^{2+}$. (b) TEM images of adsorbed $\mathrm{Pb}^{2+}$ on the pCAF/AuNC. (c) SEM image of a single pCAF/AuNC treated with 50 $\mathrm{ppm} \mathrm{Pb}^{2+}$. The adsorbed $\mathrm{Pb}^{2+}$ ions are formed as a crystal on the surface of fibers. (d) SEM image and elemental mapping of the pCAFM/AuNC treated with $50 \mathrm{ppm} \mathrm{Pb}^{2+}(\mathrm{e})$ and its EDX spectra. The inset shows the adsorbed $\mathrm{Pb}^{2+}$ crystals and their elemental mapping images. (f) XPS spectra of $\mathrm{Pb}(4 \mathrm{f})$ adsorbed on the $\mathrm{pCAFM} / \mathrm{AuNC}$.

\section{Conclusions}

To conclude, we have devised a simple and effective approach to create stable nanotraps for the effective capture of toxic metal ions in water. The obtained PCAFM/AuNC containing huge nanotraps exhibited abundant adsorption sites for metal ions, resulting in high extraction capacity up to $1587 \mathrm{mg} \mathrm{g}^{-1}$ for $\mathrm{Pb}^{2+}$. Post-encapsulation of AuNCs, the porous nature of the pCAFM/ AuNC seems to be maintained well. Interestingly, it has been found that the occurrence of pores greatly enhances the capillary effect of the pCAFM/AuNC when compared to the nCAFM/ AuNC. In addition, the facile ability of $\mathrm{Pb}^{2+}$ to penetrate into the interior of the $\mathrm{PCAF} / \mathrm{AuNC}$ enables it to form a complex with AuNC based on its availability, resulting in the formation of a crystal. This emerges as the primary reason for attaining high adsorption capacity. The comparative attribute of the pCAF/ AuNC with the nCAF/AuNC paves the way for excellent stability, porous structure and capillary effect for enhanced removal capacity. The study described here opens up a new path towards preparing a stable composite membrane containing vast nanotraps without comprising or losing the parent properties of the adsorbent.

\section{Acknowledgements}

A. S. acknowledges The Scientific \& Technological Research Council of Turkey (TUBITAK), BIDEB 2221-Fellowships for Visiting Scientists and Scientists on Sabbatical for the fellowship. B. B. acknowledges TUBITAK-BIDEB 2216, Research Fellowship Programme for Foreign Citizens for postdoctoral fellowship. A. C. acknowledges TUBITAK (project No. 113Y348) for postdoctoral funding. T. U. acknowledges FP7-Marie Curie International Reintegration Grant (IRG) for funding NANOWEB (PIRG06-GA-2009-256428). T. U. also acknowledges The Turkish Academy of Sciences - Outstanding Young Scientists Award Program (TUBA-GEBIP). Authors thank M. Guler and Dr Gokçe Çelik for TEM-STEM and ICP-MS technical support, respectively.

\section{Notes and references}

1 L. Jarup, Br. Med. Bull., 2003, 68, 167-182.

2 EPA, U.S., Drinking Water Contaminants, http:// water.epa.gov/drink/contaminants/index.cfm, accessed on 071015. 
3 M. A. Hashim, S. Mukhopadhyay, J. N. Sahu and B. Sengupta, J. Environ. Manage., 2011, 92, 2355-2388.

4 X.-F. Yu, J.-W. Liu, H.-P. Cong, L. Xue, M. N. Arshad, H. A. Albar, T. R. Sobahi, Q. Gao and S.-H. Yu, Chem. Sci., 2015, 6, 2511-2515.

5 P. A. Turhanen, J. J. Vepsäläinen and S. Peräniemi, Sci. Rep., 2015, 5, 8992.

6 M. A. Shannon, P. W. Bohn, M. Elimelech, J. G. Georgiadis, B. J. Mariñas and A. M. Mayes, Nature, 2008, 452, 301-310.

7 M. A. Barakat, Arabian J. Chem., 2011, 4, 361-377.

8 H. Y. Yang, Z. J. Han, S. F. Yu, K. L. Pey, K. Ostrikov and R. Karnik, Nat. Commun., 2013, 4, 2220.

9 I. Ali, Chem. Rev., 2012, 112, 5073-5091.

10 R. Liu, F. Liu, C. Hu, Z. He, H. Liu and J. Qu, J. Hazard. Mater., 2015, 300, 847-854.

11 B. Alies, J. D. Wiener and K. J. Franz, Chem. Sci., 2015, 6, 3606-3610.

12 D. E. Salt, M. Blaylock, N. P. Kumar, V. Dushenkov, B. D. Ensley, I. Chet and I. Raskin, Biotechnology, 1995, 13, 468-474.

13 I. Ali and V. K. Gupta, Nat. Protoc., 2006, 1, 2661-2667.

14 I. Ojea-Jiménez, X. López, J. Arbiol and V. Puntes, ACS Nano, 2012, 6, 2253-2260.

15 A. M. López_Marzo, J. Pons and A. Merkoçi, J. Mater. Chem. A, 2014, 2, 8766-8772.

16 Y. C. Shih, C. Y. Ke, C. J. Yu, C. Y. Lu and W. L. Tseng, ACS Appl. Mater. Interfaces, 2014, 6, 17437-17445.

17 J. Trujillo-Reyes, J. R. Peralta-Videa and J. L. GardeaTorresdey, J. Hazard. Mater., 2014, 280, 487-503.

18 J. Zhao, X. Zhang, X. He, M. Xiao, W. Zhang and C. Lu, J. Mater. Chem. A, 2015, 3, 14703-14711.

19 H. Ma, B. S. Hsiao and B. Chu, ACS Macro Lett., 2012, 1, 213216.

20 G. Hong, X. Li, L. Shen, M. Wang, C. Wang, X. Yu and X. Wang, J. Hazard. Mater., 2015, 295, 161-169.

21 Y. Lin, W. Cai, X. Tian, X. Liu, G. Wang and C. Liang, J. Mater. Chem., 2011, 21, 991-997.

22 D. Yang, Z. Zheng, H. Liu, H. Zhu, X. Ke, Y. Xu, D. Wu and Y. Sun, J. Phys. Chem. C, 2008, 112, 16275-16280.

23 P. Kampalanonwat and P. Supaphol, ACS Appl. Mater. Interfaces, 2010, 2, 3619-3627.

24 W. Xu, J. Wang, L. Wang, G. Sheng, J. Liu, H. Yu and X.-J. Huang, J. Hazard. Mater., 2013, 260, 498-507.

25 J. Yang, H. Zhang, M. Yu, I. Emmanuelawati, J. Zou, Z. Yuan and C. Yu, Adv. Funct. Mater., 2014, 24, 1354-1363.

26 M. X. Tan, Y. N. Sum, J. Y. Ying and Y. Zhang, Energy Environ. Sci., 2013, 6, 3254-3259.

27 L. Kong, L. Yan, Z. Qu, N. Yan and L. Li, J. Mater. Chem. A, 2015, 3, 15755-15763.

28 Y. Xie, J. Wang, M. Wang and X. Ge, J. Hazard. Mater., 2015, 297, 66-73.

29 X. Feng, G. E. Fryxell, L.-Q. Wang, A. Y. Kim, J. Liu and K. M. Kemner, Science, 1997, 276, 923-926.

30 D. Wu, F. Xu, B. Sun, R. Fu, H. He and K. Matyjaszewski, Chem. Rev., 2012, 112, 3959-4015.

31 Z. Li, D. Wu, Y. Liang, R. Fu and K. Matyjaszewski, J. Am. Chem. Soc., 2014, 136, 4805-4808.
32 L. J. Prins, Acc. Chem. Res., 2015, 48, 1920-1928.

33 C. Pezzato, S. Maiti, J. L.-Y. Chen, A. Cazzolaro, C. Gobbo and L. J. Prins, Chem. Commun., 2015, 51, 9922-9931.

34 A. Senthamizhan and T. Uyar, in Electrospinning for High Performance Sensors, ed. A. Macagnano, E. Zampetti and E. Kny, Springer International Publishing, Switzerland, 1st edn, 2015, ch. 8, pp. 179-204.

35 X.-H. Zhang, T.-Y. Zhou and X. Chen, Chin. J. Anal. Chem., 2015, 43, 1296-1305.

36 J. Sun and Y. Jin, J. Mater. Chem. C, 2014, 2, 8000-8011.

37 A. Senthamizhan, A. Celebioglu and T. Uyar, Chem. Commun., 2015, 51, 5590-5593.

38 A. Senthamizhan, A. Celebioglu and T. Uyar, J. Mater. Chem. A, 2014, 2, 12717-12723.

39 A. Senthamizhan, A. Celebioglu and T. Uyar, Sci. Rep., 2015, 5, 10403.

40 Y. Zhai, N. Wang, X. Mao, Y. Si, J. Yu, S. S. Al-Deyab, M. ElNewehy and B. Ding, J. Mater. Chem. A, 2014, 2, 14511-14518.

41 S. Anitha, B. Brabu, K. P. Rajesh and T. S. Natarajan, Mater. Lett., 2013, 92, 417-420.

42 T. Uyar, R. Havelund, J. Hacaloglu, F. Besenbacher and P. Kingshott, ACS Nano, 2010, 4, 5121-5130.

43 A. Senthamizhan, A. Celebioglu, S. Bayir, M. Gorur, E. Doganci, F. Yilmaz and T. Uyar, ACS Appl. Mater. Interfaces, 2015, 7, 21038-21046.

44 V. Thavasi, G. Singh and S. Ramakrishna, Energy Environ. Sci., 2008, 1, 205-221.

45 S. Anitha, B. Brabu, D. J. Thiruvadigal, C. Gopalakrishnan and T. S. Natarajan, Adv. Sci. Lett., 2012, 5, 468-474.

46 A. Senthamizhan, B. Balusamy and T. Uyar, Anal. Bioanal. Chem., DOI: 10.1007/s00216-015-9152-x.

47 A. Senthamizhan, B. Balusamy, Z. Aytac and T. Uyar, Anal. Bioanal. Chem., DOI: 10.1007/s00216-015-9149-5.

48 S. Anitha and T. S. Natarajan, J. Nanosci. Nanotechnol., 2013, 13, 4256-4264.

49 S. Anitha, D. J. Thiruvadigal and T. S. Natarajan, Mater. Lett., 2011, 65, 2872-2876.

50 Z. M. Huang, Y. Z. Zhang, M. Kotaki and S. Ramakrishna, Compos. Sci. Technol., 2003, 63, 2223-2253.

51 L. Persano, A. Camposeo, C. Tekmen and D. Pisignano, Macromol. Mater. Eng., 2013, 298, 504-520.

52 W. E. Teo and S. Ramakrishna, Nanotechnology, 2006, 17, R89-R106.

53 R. Sahay, P. S. Kumar, R. Sridhar, J. Sundaramurthy, J. Venugopal, S. G. Mhaisalkar and S. Ramakrishna, J. Mater. Chem., 2012, 22, 12953-12971.

54 D. Kai, S. S. Liow and X. J. Loh, Mater. Sci. Eng., C, 2014, 45, 659-670.

55 S. Cavaliere, S. Subianto, I. Savych, D. J. Jones and J. Rozière, Energy Environ. Sci., 2011, 4, 4761-4785.

56 K. A. Rieger, N. P. Birch and J. D. Schiffman, J. Mater. Chem. $B, 2013,1,4531-4541$.

57 A. Celebioglu and T. Uyar, Mater. Lett., 2011, 65, 2291-2294.

58 H. Ding, C. Liang, K. Sun, H. Wang, J. K. Hiltunen, Z. Chen and J. Shen, Biosens. Bioelectron., 2014, 59, 216-220.

59 A. Senthamizhan, A. Celebioglu, B. Balusamy and T. Uyar, Sci. Rep., 2015, 5, 15608. 
60 A. Kr zel, W. Lesniak, M. Jezowska-Bojczuk, P. Mlynarz, J. Brasuñ, H. Kozlowski and W. Bal, J. Inorg. Biochem., 2001, 84, 77-88.

61 A. Gupta, A. Chaudhary, P. Mehta, C. Dwivedi, S. Khan, N. C. Verma and C. K. Nandi, Chem. Commun., 2015, 51, 10750-10753.

62 A. Chaudhary, C. Dwivedi, M. Chawla, A. Gupta and C. K. Nandi, J. Mater. Chem. C, 2015, 3, 6962-6965.

63 J. Zhang, Y. Yuan, G. Liang, M. N. Arshad, H. A. Albar, T. R. Sobahi and S.-H. Yu, Chem. Commun., 2015, 51, 10539-10542.

64 J. A. Prince, D. Rana, T. Matsuura, N. Ayyanar, T. S. Shanmugasundaram and G. Singh, Sci. Rep., 2014, 4, 6949.

65 M. Tao, L. Xue, F. Liu and L. Jiang, Adv. Mater., 2014, 26, 2943-2948.

66 J. J. Alcaraz-Espinoza, A. E. Chávez-Guajardo, J. C. MedinaLlamas, C. A. S. Andrade and C. P. de Melo, ACS Appl. Mater. Interfaces, 2015, 7, 7231-7240.

67 J. Yuan, X. Liu, O. Akbulut, J. Hu, S. L. Suib, J. Kong and F. Stellacci, Nat. Nanotechnol., 2008, 3, 332-336.
68 W. Mai, B. Sun, L. Chen, F. Xu, H. Liu, Y. Liang, R. Fu, D. Wu and K. Matyjaszewski, J. Am. Chem. Soc., 2015, 137, 1325613259.

69 M. Lejuene, L. M. Lacroix, F. Bretagnol, A. Valsesia, P. Colpo and F. Rossi, Langmuir, 2006, 22, 3057-3061.

70 S. Anitha, B. Brabu, D. J. Thiruvadigal, C. Gopalakrishnan and T. S. Natarajan, Carbohydr. Polym., 2013, 97, 856-863.

71 X. Lu, J. Zhou, Y. Zhao, Y. Qiu and J. Li, Chem. Mater., 2008, 20, 3420-3424.

72 A. Li, H.-X. Sun, D.-Z. Tan, W.-J. Fan, S.-H. Wen, X.-J. Qing, G.-X. Li, S.-Y. Li and W.-Q. Deng, Energy Environ. Sci., 2011, 4, 2062-2065.

73 M. Guo, B. Ding, X. Li, X. Wang, J. Yu and M. Wang, J. Phys. Chem. C, 2010, 114, 916-921.

74 C. Xiao, X. Zhang, J. Liu, A. Yang, H. Zhao, X. Li, Y. He and Z. Yuan, Anal. Methods, 2015, 7, 924-929.

75 J. Y. Kim and J. S. Lee, Nano Lett., 2009, 9, 4564-4569.

76 D.-H. Tsai, T. J. Cho, F. W. DelRio, J. M. Gorham, J. Zheng, J. Tan, M. R. Zachariah and V. A. Hackley, Langmuir, 2014, 30, 3397-3405. 